for the establishment of an East African Marine Fisheries Research Survey in Zanzibar and $£ 53,000$ for the establishment of a West African Institute of Social and Economic Research. The establishment of a Fisheries Research Organization to serve Northern Rhodesia and Nyasaland receives $£ 62,000$, and $£ 55,000$ is provided for the establishment of headquarters in Tanganyika of the East African Insecticides Research Unit, $£ 65,000$ for experiments with the dissemination of insecticides from fixed-wing aircraft, $£ 31,912$ for research on bananas, soils, cocoa, and sugar technology at the Imperial College of Tropical Agriculture, a further $£ 30,300$ for the establishment of the Colonial Microbiological Research Institute, $£ 47,000$ for the formation of a filariasis research unit in East Africa, $£ 19,500$ for establishing an East African Malarial Unit, $£ 10,500$ for the construction and equipment of an East African Herbarium at Nairobi, and $£ 16,616$ for the establishment of a Tsetse Fly Research Unit in Northern Rhodesia. Supplementary capital provision for the East African Agriculture and Forestry and Veterinary Research Organizations amounts to $£ 21,000$; besides $£ 14,955$ for the investigation of the incidence of loiasis in the British Cameroons, $£ 9,300$ is provided for capital expenditure on research on the incidence of loiasis in Nigeria. Hot-climate physiology research in Nigeria receives $£ 11,839$, fundamental insecticides

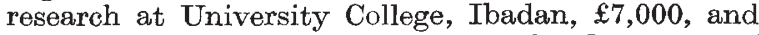
the establishment and maintenance for five years of an Institute of Educational Research to serve Fiji, the British Solomon Islands Protectorate and the Gilbert and Ellice Islands, £21,017. A grant of $£ 18,813$ is for assistance towards the expansion and operation of the Elini Livestock Experimental Station, British Guiana, and one of $£ 17,600$ for research to ascertain causes of the swarming of the Moroccan locust and the best means of prevention; $£ 20,000$ is provided for the formation of a small pool of entomologists to undertake short-term investigations in the Colonies, especially in smaller territories which have no government entomologists on their establishments. Other miscellaneous grants provide for six postgraduate studentships for two years training in insecticides research, for fundamental research on Colonial insecticide problems at Rothamsted Experimental Station and at the Field Station of the Imperial College of Science and Technology, for Colonial medical research studentships, for the appointment of Colonial liaison officers at the Pest Infestation Laboratory and the Road Research Laboratory of the Department of Scientific and Industrial Research, and for research at the Lister Institute of Preventive Medicine into the preparation of precipitin sera for entomological investigations of malaria, trypanosomiasis, etc.

Nor does this indicate the full extent of the demands now being made by the Colonial Development and Welfare Schemes on the resources of Great Britain both in finance and in man-power. Besides the schemes listed specifically as research schemes, such general schemes as the desert locust survey and emergency control measures and the establishment of a malarial unit must also make appreciable demands on scientific man-power and on finance. Only a few of such schemes can be mentioned here, but the development plan for Uganda for which $£ 697,000$ is allotted, the control of 'sudden death' disease of cloves in Zanzibar $(£ 193,000)$, the livestock diseases survey in Nyasaland $(£ 39,970)$, geological surveys in Northern

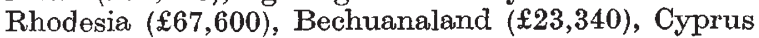

$(£ 46,000)$, North Borneo and Sarawak (£107,650), Gold Coast $(£ 21,200)$, the control of yaws in Jamaica $(£ 45,512)$, tsetse control in Northern Nigeria ( $\$ 355,960)$, the development of water supplies in Malta ( $(5536,800$ ), are schemes which sufficiently illustrate the variety and magnitude of the demand they make on the resources of Great Britain. This is quite apart from what is involved in such grants for higher educational development as that of $£ 332,821$ for the first stage of development of the permanent University College at Ibadan, Nigeria, the miscellaneous grants for the University College of the West Indies, including $£ 207,000$ for the construction of permanent buildings, $£ 565,834$ for the construction and equipment of a new technical college for the Federation of Malaya, $£ 82,917$ for an agricultural experimental station at Trinidad, and the $£ 58,638$ for completing the building programme of the Imperial College of Tropical Agriculture. The numerous grants for the Colonial Service training schemes, for agricultural development, fisheries development, forestry, road development and the Caribbean Medical Centre and the Suva Medical Centre, Fiji, all likewise represent a considerable contribution both of scientific manpower and of financial resources the magnitude of which is probably not generally appreciated, but may well bring a rich return to Great Britain as well as to the Colonial peoples.

\section{GOLD MEDAL OF THE ROYAL ASTRONOMICAL SOCIETY}

\section{AWARD TO Prof. J. STEBBINS}

T AST year the Gold Medal of the Royal Astron1 omical Society, which had been awarded to Prof. Joel Stebbins, was presented to Dr. C. S. Piggott, scientific attaché to the American Embassy in London, acting as proxy for Prof. Stebbins, who was unable to be present. Prof. W. M. Smart, president of the Society, delivered a presentation address which described the work of Prof. Stebbins and his specialization in photometric observations of practically every class of celestial bodies except meteors and planetary nebulæ. This address by the president has been published in full (Mon. Not. Rcy. Astro. Soc., 110, 2, 179; 1950).

Soon after going to Urbana Observatory in the University of Illinois in 1903, Prof. Stebbins commenced experiments with selenium cells, some of which he made himself, but the Dutch firm of Giltay, at Delft, produced the most satisfactory one. The first successful observations were made on the moon, and Prof. Stebbins found that the light of full moon is 0.22 candle-metres. At half-moon during the first quarter, the brightness is only 12 per cent of that of the full moon, and at last quarter it is reduced to 10 per cent. This is due to the irregularities of the lunar surface, the dark seas covering a large portion of the sunlit area during the last quarter. During a partial eclipse of the moon, he found that the instant of minimum light took place within $0.6 \mathrm{~min}$. of the time predicted by the ephemeris. The selenium cell was also used to determine the light-curve of Algol, with very satisfactory results. For the first time the secondary minimum was observed, and the discovery at the same time of a continuous variation on either side of the secondary minimum suggested that the 
fainter component $B$ has an axial period of rotation equal to its orbital period of revolution with respect to the brighter component $A$. In addition, the hemisphere of $B$ exposed to the radiation from $A$ is brighter than the other hemisphere; this was the first time that this 'reflexion effect' had been observed. When Stebbins took up the study of Algol ten years later, using the more accurate photo-electric photometer, not only were the general features of the binary as determined with the selenium cell confirmed, but also another effect arising from the slightly ellipsoidal shape of the components was detected.

Algol, however, was one of the exceptions to Eddington's mass-luminosity relation on the assump. tion that the mass of $A$ was 0.37 that of the sun and the mass of $B$ half that of $A$. Later, when McLaughlin and Rossiter, using a new method, applied it to the solution of the binary problem, it was found that $A$ was five times as massive as $B$, and $A$ then satisfies the mass-luminosity requirements, thus vindicating the accuracy and importance of Stebbins's work.

At the total solar eclipses of 1918, 1925 and 1937, Stebbins measured the integrated light of the corona with his photo-electric apparatus, and concluded that there is little evidence of any noticeable variation of the total light of the corona during the sunspot period. Another interesting result was that the light of the corona, photo-electrically, is about one-half that of the full moon. From the absence of evidence of the Rayleigh scattering, it was inferred that the continuous and Fraunhofer spectrum can be produced by electrons or large dust particles, but not by atoms, molecules and particles of wave-length dimensions. Finally, over a large range of wave-lengths the intensity distributions for the sun and corona are the same- a fact which must be taken into consideration by all who formulate any general theory of the corona.

It is impossible to refer to all the branches of Stebbins's work, and it will be sufficient to mention one or two others of outstanding importance. In 1930, when he visited Lick Observatory, he turned his attention to an extensive observational programme on stars (starting with $B$ stars), globular clusters and extragalactic nebulæ. This programme was so comprehensive and so important that it is well worth reading Prof. Smart's address to realize the implications of some of the results obtained. It may be mentioned that the evidence obtained from the globular clusters led to a revision not only of their individual distances but also of the diameter of the galaxy, which was shown to be considerably less than that of Shapley's original estimate and is now believed to be about 100,000 light-years or less. This reduction in the dimensions of our galaxy, combined with Stebbins's work on the Andromeda nebula with the photo-electric observations of its outlying regions, which showed it to be considerably larger than was originally believed, has led to the conclusion that there is little difference in the overall dimensions of the two systems.

In 1947 Stebbins attempted to locate the central nucleus of the galaxy. Using the 60-inch telescope at Mt. Wilson, the galvanometer deflexions for the infra-red filter $(10,300 \mathrm{~A}$.) and also for a red filter (7,190 A.) were noted in narrow zones, with the telescope stationary, in the neighbourhood of $326^{\circ}$ galactic longitude. The observations extended over several degrees in longitude and latitude, and the maxima of the infra-red readings appeared to indicate a bulge. An analysis of the space-reddening showed that the light of the bulge, extending over $8^{\circ}$ in longitude and $4-5^{\circ}$ in latitude, when corrected for absorption, is comparable with the light of an equivalent section of the Andromeda nebula. The position, outline and total light of the bulge suggest that something is being detected near the galactic centre, but Stebbins makes no greater claim than this.

Prof. Stebbins's work is well summed up at the end of Prof. Smart's address : "In surveying our Medallist's work over the long period of nearly fifty years one cannot but be impressed by his devotion to one great department of astronomy which he in great measure has made his own, by his observational skill, his attention to detail and his critical analysis of the new and unexpected phenomena which his observations have brought to light".

\section{INSTITUTION OF GAS ENGINEERS}

\section{AUTUMN RESEARCH MEETING}

$T$ HE sixteenth autumn research meeting of the Institution of Gas Engineers was held in London during November 28-29, 1950, with Mr. F. M. Birks as president.

Like other public services, the gas industry has problems of peak load, if of less intensity. Normally they are met by the use of carburetted water gas, a product which makes the combined use of gasified coke and mineral oil. H. Stanier and J. B. McKean described a new process based on the gasification of mineral oil, blended with steam and heated in contact with a suitable catalyst. Under suitable conditions the product is a gas of composition and character similar to normal town's gas, and can be used for supplementing the latter at peak periods. From economic considerations this process might provide an alternative to gas manufactured from coal.

At the present time, the sulphur available from home sources is obtained by the purification of coal gas. The normal methods of recovery, although efficient, have certain practical difficulties, and any improved means might be an important contribution to the national economy. E. Johnson described an attempt to recover sulphur of high purity from the spent oxide of gas purification practice using a fluidized technique and solvent extraction. A sulphur product of 95 per cent purity is obtained, and the extracted oxide has a reactivity to hydrogen sulphide not greatly different from that of the original material. The work has shown that a semi-continuous process for the extraction of sulphur from spent oxide in a fluidized bed by means of solvents is possible, and no fundamental difficulties are likely to be encountered in making the process fully continuous. A large-scale fluidized process was proposed for further investigations.

The importance of combustion research in gas utilization was emphasized by J, E. Garside in a review of the field of relevant research. Flame propagation, flame stability, mechanism of combustion reactions, heat and energy release, heat and energy transfer, and ignition of gas-air mixtures were all briefly dealt with, and the extent of present-day knowledge indicated. Some of the major questions facing gas utilization in the industry at the present time were stated, and an attempt was made to answer them by explaining the findings of current researches in Great Britain and correlating those findings with the results of similar projects overseas. 